\title{
Severity of obstructive sleep apnea and metabolic variables: complex relationship needs comprehensive consideration
}

\author{
Jianrong Hu $\cdot$ Chunlin Tu $\cdot$ Jingxian Sun $\cdot$ \\ Yilin Wang
}

Received: 2 June 2014 / Accepted: 15 June 2014/Published online: 3 July 2014

(C) Springer-Verlag Berlin Heidelberg 2014

\section{Dear Editor:}

We recently read the article named "Correlation between the severity of apnea and hypopnea sleep, hypertension and serum lipid and glycemic: a case control study" conducted by de Sousa Rodrigues and Lira [1] with great interest. In this well-presented study, the authors explored the correlation between the severity of obstructive sleep apnea (OSA) and the levels of blood pressure (BP), lipids and glucose; however, no correlations between the severity of OSA and metabolic variables were found.

First of all, we congratulate the authors for carrying out such an important study of the severity of OSA and hypertension, dyslipidemia and hyperglycemia. However, the conclusion of this study differs from previous studies focused on increased BP, lipid profile, insulin resistance and respiratory disturbance index [2-4]. This inconsistence may partly be explained by the small sample size in this study, which should be estimated through power calculations (a two-sided confidence level of $99.9 \%$ and power of $80 \%$ ). 6-11 subjects per group may not be sufficient to draw a conclusion.

Besides, some additional considerations should be mentioned. First, physical activity and dietary habits may be associated with metabolic disorders in OSA patients [5], thus these factors should be considered. In addition, confounding effects of gender difference and medications may also have influence in metabolic variables, and these factors should also be taken into account. Finally, inflammatory cytokines (i.e., interleukins, tumor necrosis factor- $\alpha$, and C-reactive protein) and adipokines (i.e., leptin,

J. Hu $(\bowtie) \cdot$ C. Tu $\cdot$ J. Sun $\cdot$ Y. Wang

Department of Respiratory Medicine, Jiading Central Hospital,

1 Chengbei Road, Shanghai 201800, China

e-mail: jianronghu@126.com adiponectin, visfatin and resistin) are related to obesity, hypertension and apnea [6]. These biomarkers altered in metabolic conditions with OSA.

The results might be more convincible if the authors enroll more participants and give information about the above-mentioned factors. We believe that these findings will provide valuable information to clarify the comprehensive relationship between metabolic variables and the severity of OSA.

Conflict of interest The authors have reported that no potential conflicts of interest exist with any companies/organizations whose products or services may be discussed in this article.

\section{References}

1. de Sousa Rodrigues CF, Lira AB (2014) Correlation between the severity of apnea and hypopnea sleep, hypertension and serum lipid and glycemic: a case control study. Eur Arch Otorhinolaryngol. (Epub ahead of print)

2. Muxfeldt ES, Margallo VS, Guimarães GM et al (2014) Prevalence and associated factors of obstructive sleep apnea in patients with resistant hypertension. Am J Hypertens. (Epub ahead of print)

3. Toyama Y, Chin K, Chihara Y et al (2013) Association between sleep apnea, sleep duration, and serum lipid profile in an urban, male, working population in Japan. Chest 143:720-728

4. Punjabi NM, Sorkin JD, Katze LI et al (2002) Sleep-disordered breathing and insulin resistance in middle-aged and overweight men. Am J Crit Care Med 165:677-682

5. Romero-Corral A, Caples SM, Lopez-Jimenez F et al (2010) Interactions between obesity and obstructive sleep apnea: implications for treatment. Chest 137:711-719

6. Rasouli N, Kern PA (2008) Adipocytokines and the Metabolic Complications of Obesity. J Clin Endocrinol Metab 93(11 Suppl 1):S64-S73 